\title{
FASCE, JORGE (2011). DIRIGIR UNA ESCUELA: UNA VISIÓN INTEGRAL. BUENOS AIRES: 12NTES
}

\author{
Lorena Natalia Plesnicar (Universidad Nacional de La Pampa** \\ lorena.plesnicar@speedy.com.ar
}

Recibido: 5/07/2012 Aceptado: 29/08/2012

En los últimos años, la preocupación sobre la dirección de las instituciones educativas atraviesa tanto a los responsables de las políticas públicas, a quienes ejercen los cargos en las escuelas como así también a numerosos investigadores/as que se dedican a su estudio desde distintos posicionamientos teóricos, metodológicos y epistemológicos.

En este sentido, el libro Dirigir una escuela: una visión integral propone una aproximación al tema desde la constante interpelación de la teoría y la práctica. En el primer capítulo denominado "El director de escuela" el autor plantea desde la aceptación de la complejidad de la escuela los alcances y límites de la acción directiva. En esta línea, recurre a la distinción entre "dirigir", "gestionar", "coordinar" y "gobernar" para argumentar después la necesidad de revisar los conceptos de poder y autoridad y sus implicancias en el quehacer cotidiano.

El asunto de la comunicación en la escuela desde la función directiva es el eje que articula el segundo capítulo del libro. Jorge Fasce plantea la relevancia de analizar el entramado de relaciones que acontecen en las instituciones y el lugar del director en los procesos comunicativos. En su perspectiva, se trata de que los directores tomen la decisión estratégica de sostener un sistema de comunicación en un encuadre específico que posibilite tanto el desarrollo de las tareas institucionales como el desarrollo profesional de los docentes.

Recuperar el rol pedagógico de los directores es la propuesta de Fasce para mejorar la conducción integral de las instituciones educativas y este es el tema central sobre el que versa el capítulo 3 del libro. En esta línea, su apuesta

* Doctora en Ciencias Sociales (FLACSO). Mg. en Dirección y Gestión de Centros Educativos (Universidad de Barcelona). Profesora de Ciencias de la Educación (Universidad Nacional de La Pampa). Profesora de Nivel Inicial (Universidad Nacional de La Pampa). 
teórica se sostiene en la relevancia del director de escuela en la formación de los docentes. En otras palabras, que los directores reconozcan y asuman su rol enseñante es prioritario para pensar la conducción pedagógica del cargo de dirección.

"El director y sus tareas cotidianas" es el título del capítulo cuatro del libro. El mismo se inicia con algunas reflexiones sintéticas sobre la elaboración del Proyecto Educativo Institucional. Para el autor la decisión por los modos de formular un PEI (centrada en el equipo de conducción o en la que participen todos los actores de la institución) puede marcar el desarrollo del mismo. Otro aspecto también abordado en el capítulo es sobre la función de coordinación del director tanto en el equipo de conducción como con el equipo docente. A esto se suma una síntesis sobre prácticas referidas a las reuniones de personal y con las familias.

El asunto de la evaluación es el eje que recorre el capítulo quinto. En esta línea, se describe una propuesta de evaluación integral de la escuela que contempla desde el planteamiento ético de la evaluación, la coevaluación, la observación de clases para la evaluación de los docentes hasta la autoevaluación del director para mejorar su tarea profesional.

El libro concluye con un último capítulo en el que se plantean numerosos interrogantes sobre las cualidades personales y profesionales del director. Este debate que ha ya sido planteado en numerosos estudios -por ejemplo, sobre si las cualidades personales de un director son innatas o aprendidas o sobre la relevancia del carisma y del liderazgo- aquí se propone como cierre del recorrido sobre la reflexión de dirigir una escuela desde una visión integral.

Para finalizar esta reseña quiero hacer dos comentarios sobre el libro. El primero es aclarar que el texto contiene numerosas propuestas de evaluación porque plantea a los docentes la opción de tomarlo como un curso, para lo cual se adjuntan dos DVD's como recursos para acompañar la propuesta de formación docente continúa. El segundo es sobre la mirada misma que propone el autor acerca de la dirección de las instituciones educativas. En efecto, y como anunciamos al inicio, aunque es un texto que promueve la articulación constante entre escenas cotidianas de las instituciones y aportes teóricos obvia en forma continua la inscripción del tema en un encuadre que dialogue con las políticas educativas y sus implicancias en los complejos escenarios institucionales actuales. 